International Journal of Canadian Studies

Revue internationale d'études canadiennes

\title{
Une politique du bien commun au Canada est-elle possible?
}

\section{Stéphane Courtois}

Numéro 42, 2010

URI : https://id.erudit.org/iderudit/1002182ar

DOI : https://doi.org/10.7202/1002182ar

Aller au sommaire du numéro

\section{Éditeur(s)}

Conseil international d'études canadiennes

\section{ISSN}

1180-3991 (imprimé)

1923-5291 (numérique)

Découvrir la revue

Citer cet article

Courtois, S. (2010). Une politique du bien commun au Canada est-elle possible? International Journal of Canadian Studies / Revue internationale d'études canadiennes, (42), 273-282. https://doi.org/10.7202/1002182ar
Résumé de l'article

Mon texte entend offrir un commentaire critique de l'ouvrage de Charles Blattberg Et si nous dansions? Pour une politique du bien commun au Canada (2004). Il entend plus particulièrement examiner la conception, qualifiée de "patriotique» et axée sur la "conversation", que se fait Blattberg de la pratique du dialogue, pratique dont il attend qu'elle favorise le rapprochement des différentes communautés nationales (canadienne-anglaise, québécoise et amérindiennes) dont se compose le Canada. L'une des particularités de cette conception est son opposition à une variante importante de l'approche dialogique au sein de la philosophie politique contemporaine représentée par la démocratie délibérative. Mon texte se propose d'examiner le bien-fondé de cette opposition. Après avoir rappelé les grands traits de l'approche patriotique et conversationnelle de Blattberg, mon texte examine en détail les raisons de son opposition à l'approche délibérative pour, finalement, démontrer que l'approche patriotique risque de poser des difficultés plus grandes que celles qu'elle entend résoudre. 


\title{
Stéphane Courtois
}

\section{Une politique du bien commun au Canada est-elle possible?}

\section{Résumé}

Mon texte entend offrir un commentaire critique de l'ouvrage de Charles Blattberg Et si nous dansions? Pour une politique du bien commun au Canada (2004). Il entend plus particulièrement examiner la conception, qualifiée de "patriotique» et axée sur la "conversation», que se fait Blattberg de la pratique du dialogue, pratique dont il attend qu'elle favorise le rapprochement des différentes communautés nationales (canadienne-anglaise, québécoise et amérindiennes) dont se compose le Canada. L'une des particularités de cette conception est son opposition à une variante importante de l'approche dialogique au sein de la philosophie politique contemporaine représentée par la démocratie délibérative. Mon texte se propose d'examiner le bienfondé de cette opposition. Après avoir rappelé les grands traits de l'approche patriotique et conversationnelle de Blattberg, mon texte examine en détail les raisons de son opposition à l'approche délibérative pour, finalement, démontrer que l'approche patriotique risque de poser des difficultés plus grandes que celles qu'elle entend résoudre.

\begin{abstract}
My text is a critical commentary of Charles Blattberg's work Et si nous dansions? Pour une politique du bien commun au Canada (2004). More specifically, I examine Blattberg's conception of dialogue, qualified as "patriotic" and centered on "conversation", and which he believes favours the reconcilement of different national communities that make up Canada (Anglo-Canadian, Québécois, and Amerindian). One of the particularities of Blattberg's thought is its opposition to an important variant of the dialogic approach in contemporary political philosophy as represented by deliberative democracy. My text examines the validity of this opposition. After drawing the broad strokes of Blattberg's patriotic and conversational approach, I examine in detail the reasons for its opposition to the deliberative approach, and finally I demonstrate that the patriotic approach risks raising difficulties that are greater than those it aims to resolve.
\end{abstract}

Dans un livre récent intitulé Et si nous dansions? Pour une politique du bien commun au Canada (2004), Charles Blattberg propose une conception des plus intéressantes de la situation politique au Canada, de ses maux et de la façon de les surmonter. La question directrice de son livre consiste à savoir pourquoi nous nous sentons si peu « chez nous » dans le paysage constitutionnel de notre pays. Il impute cette situation aux politiques mises en avant sous l'ère de Pierre Elliott Trudeau, lesquelles, inspirées d'une théorie 
neutraliste de l'État et d'une théorie individualiste de la justice, auraient contribué à rendre invisibles les différentes communautés nationales dont se compose le Canada. Or, selon Blattberg, il existerait au Canada une véritable communauté nationale canadienne-anglaise, au même titre qu'il existerait une communauté nationale québécoise et plusieurs autres communautés nationales amérindiennes. Ces nations, cependant, devraient être distinguées de la communauté civique, c'est-à-dire de l'État central, siège d'une citoyenneté commune et d'une culture politique distincte des diverses cultures nationales. Son rôle, cependant, ne devrait pas se limiter à coordonner les relations des citoyens entre eux en les protégeant au moyen de droits individuels, erreur commise selon Blattberg sous le règne de Trudeau. Le rôle de l'État central devrait plutôt être de favoriser la poursuite du bien commun, qui serait source d'un véritable patriotisme. Selon Blattberg, ce bien commun ne pourra toutefois voir le jour que si les membres des diverses cultures nationales s'efforcent de surmonter leurs différends par la pratique du dialogue et la recherche d'une véritable compréhension mutuelle, condition jugée indispensable à leur adhésion patriotique à la communauté politique plus large.

J'entends dans ce texte examiner plus précisément la façon dont Blattberg conçoit cette pratique du dialogue dont dépendraient selon lui le rapprochement des différentes communautés dont se compose le Canada et l'avenir de la fédération canadienne elle-même. La pratique du dialogue correspond chez Blattberg à une certaine approche de la politique qu'il nomme " patriotique » et qui serait axée sur la « conversation ». Mais il existe plusieurs approches, au sein de la philosophie politique contemporaine, qui insistent aussi sur le dialogue et la conversation comme mode de résolution des conflits, et Blattberg tient à opposer la variante patriotique qu'il défend à l'une des variantes actuellement les plus influentes, représentée par la démocratie délibérative. J'entends discuter du bien fondé de cette opposition. Je procéderai en trois temps. Je rappellerai tout d'abord en quoi consiste exactement l'approche patriotique de la politique défendue par Blattberg. J'examinerai ensuite les raisons de son opposition à l'approche délibérative. Finalement, je montrerai que l'approche patriotique de Blattberg risque de présenter des difficultés plus grandes que celles qu'elle entend surmonter, rendant plus qu'incertaine une politique du bien commun au Canada.

\section{I}

Deux caractéristiques essentielles permettent de cerner l'approche patriotique de la politique que préconise Blattberg. La première consiste à considérer l'État et ses institutions comme l'expression d'un « bien commun ». Il convient ici d'attirer l'attention sur les deux termes, «bien » et « commun ». Un « bien » est pour Blattberg (Et si nous dansions 84 ) une chose à laquelle on accorde de la valeur. Ce bien peut avoir une valeur instrumentale, s'il a une utilité extérieure à lui-même, comme l'argent, ou une valeur intrinsèque, s'il est 
un bien en lui-même, comme la liberté ou la dignité des personnes. Les biens sont « communs » s'ils sont partagés par plusieurs individus. Ainsi, un barrage qui alimente une ville en eau est un exemple de bien commun instrumental, tandis que l'amitié s'offre comme un exemple de bien commun intrinsèque. Blattberg qualifie les biens communs de "publics » lorsqu'ils sont partagés par un très grand nombre d'individus formant des communautés. La nation et l'État sont pour lui des exemples de biens communs publics ayant une valeur intrinsèque. Cependant, contrairement aux nationalistes qui ont tendance à identifier « nation » et « État », Blattberg considère les communautés nationales comme des réalités sociologiques qu'il localise, non au niveau de l'État, mais de la société civile (86) ${ }^{1}$. Le patrimoine commun (langue, productions culturelles, histoire), et la culture nationale qui s'y exprime, seraient le type de bien commun propre à ces communautés. L'État et ses institutions, ainsi que la culture politique qu'ils incarnent, seraient de leur côté l'expression d'un bien commun sui generis, celui de la citoyenneté, qui serait «l'essence de ce qui fait d'un pays un tout » (94), une véritable communauté politique ou civique. Blattberg considère cette communauté plus large de manière holistique : elle formerait un tout organique, une « forme de vie» faite d'une diversité de communautés (non seulement nationales, mais également ethniques, religieuses et régionales, en plus des associations civiles), elle serait en quelque sorte une " communauté de communautés », dont l'intégrité et la survie dépendraient de la capacité du tout à évoluer en s'adaptant aux parties et, réciproquement, de la capacité des parties à s'ajuster au tout (39-40). Les sources d'inspiration de Blattberg vont nettement ici, de son propre aveu, du côté de courants comme la philosophie herméneutique (55).

La seconde caractéristique de l'approche patriotique de la politique de Blattberg se situe au plan des moyens jugés les plus appropriés à la réalisation du bien commun au plan civique et politique. L'État et ses institutions ne pourront prétendre incarner un véritable bien commun pour les citoyens ayant à leurs yeux une valeur intrinsèque et faisant l'objet d'un authentique patriotisme que s'ils sont disposés à faire usage de leurs libertés politiques et de leurs vertus civiques en vue de concilier leurs différends. Blattberg puise ici son inspiration dans des idées familières à la tradition du républicanisme classique, allant d'Aristote à Hannah Arendt, mais il se démarque dans sa façon d'envisager les vertus civiques attendues des citoyens et l'exercice de leurs libertés politiques, qu'il conçoit en termes communicationnels ou dialogiques : c'est en effet, non simplement par la recherche de compromis négociés, mais par celle d'une véritable compréhension mutuelle, par la pratique du dialogue, plus précisément, par la pratique de la « conversation » (40), qu'il attend des citoyens qu'ils résolvent leurs conflits et poursuivent le bien commun. Pour être en mesure d'apprécier ce qui appartient en propre à l'approche patriotique de Blattberg, j'aimerais rappeler brièvement ce qui la distingue, d'une part, des approches non-conversationnelles de la politique 
et, d'autre part, des autres approches de la politique préconisant le recours au dialogue comme mode de résolution des conflits.

Blattberg oppose son approche patriotique à deux autres approches de la politique qui seraient selon lui peu sensibles au dialogue et à la conversation. Il qualifie la première de "monarchiste » (19-26). Selon cette dernière, il ne pourrait y avoir de justice que sous une autorité souveraine qui ne souffre aucune contestation ou remise en question. Le constitutionnalisme juridique, forme de réglementations des différends ayant de plus en plus imprégné la politique canadienne depuis l'ère Trudeau, où les conflits sont tranchés par une autorité représentée par la Cour suprême, serait un exemple de l'approche monarchiste de la politique que condamne Blattberg sans ambages (23-4). La seconde approche de la politique est qualifiée de polyarchiste (27-30). À la différence de la première, celle-ci mettrait davantage l'emphase sur la pluralité que sur l'unité. Selon ses défenseurs, il existerait trop de visions du monde contradictoires dans nos sociétés pluralistes pour qu'une seule conception de la justice puisse faire autorité. Alors que les tenants d'une version davantage " adversative » du polyarchisme verraient la politique comme le lieu d'affrontement perpétuel entre ennemis où la loi du plus fort règne, pour les tenants d'une version plus modérée du polyarchisme, qualifiée de " pluraliste ", la recherche de compromis et d'arrangements stratégiques par le biais de la négociation serait un moyen plus adéquat de résolution des conflits (27-30). Mais pour Blattberg, quelle que soit la version du polyarchisme qui est mise en avant, cette approche de la politique partagerait avec le monarchisme le même défaut : ne donner aucun droit de cité au dialogue, à la compréhension mutuelle, à la « conversation » comme mode de résolution des différends, puisque les compromis ne mènent au mieux qu'à la conciliation d'intérêts divergents, non au rapprochement des interlocuteurs que rendrait possible un véritable dialogue.

L'approche patriotique de Blattberg s'oppose cependant, non seulement aux approches non-conversationnelles de la politique que je viens de mentionner, mais aussi à d'autres approches faisant de la conversation ou du dialogue entre les membres d'une communauté politique le fondement de la résolution des conflits. Ce que Blattberg reproche à ces autres variantes de l'approche conversationnelle, c'est, ou bien de faire une distinction trop tranchée entre « conversation » et « négociation », ce qui les rapprocherait dangereusement de l'approche monarchiste, ou bien de confondre tout simplement les deux, ce qui les rapprocherait cette fois de l'approche polyarchiste (43-44). Je me concentrerai dans ce qui suit sur la variante de l'approche conversationnelle représentée par la démocratie délibérative, que Blattberg critique sévèrement et à laquelle il oppose sa propre variante patriotique. 
Blattberg a plusieurs reproches à adresser à la démocratie délibérative. Les premiers, plus spécifiques, touchent aux règles de la discussion que recommandent les démocrates délibératifs (Blattberg, "Patriotic » 156-158). Jürgen Habermas, dont les travaux représentent la cible principale des critiques de Blattberg, propose trois groupes de règles. Les premières sont syntaxiques et sémantiques : les locuteurs doivent s'efforcer d'utiliser les prédicats que contiennent leurs énoncés de manière consistante; ils doivent, en d'autres termes, éviter de commettre des contradictions ou d'accorder différents sens à une même expression (Habermas, Morale et communication 108-109). Mais ces règles nous interdisent alors, selon Blattberg ( Patriotic »157), de faire de l'humour, des jeux d'esprit, ou d'utiliser des métaphores, lesquels peuvent pourtant contribuer à rapprocher les partis en conflit. Le second groupe de règles vise à assurer la sincérité des délibérants : les locuteurs doivent soutenir uniquement ce qu'ils croient réellement être vrai; ils ne doivent donc pas chercher à tromper les autres sur leurs intentions ou leurs croyances véritables (Habermas, Morale et communication 109). Mais alors, estime Blattberg («Patriotic »157), cela témoigne de l'incapacité de Habermas à apprécier des choses telles que la rhétorique, la flatterie, l'exagération, voire les mensonges discrets, qui peuvent aussi contribuer à réconcilier les partis en conflit. Le troisième groupe de règles, les plus importantes pour Habermas et les démocrates délibératifs, vise à assurer l'inclusion de tous les participants, de même que l'égalité des chances des interlocuteurs : aucun locuteur compétent ne peut être exclu du discours; tous doivent avoir les mêmes chances de faire valoir leurs perspectives d'interprétation, leurs besoins et leurs intérêts; et aucune contrainte, externe ou interne, autre que celle, rationnelle, du meilleur argument ne peut être admise au sein de la discussion (Habermas, Morale et communication 110-111). Cependant, ces règles sont pour Blattberg beaucoup trop utopiques («Patriotic » 158). L'exigence d'inclusion de tous les participants, jointe au principe d'universalisation recommandant de n'admettre comme valables que les normes exprimant des intérêts généralisables, implique une forme de cosmopolitisme qui s'avère incompatible avec le fait de n'admettre aux discussions que nos concitoyens. (158) Elle est également incompatible avec le fait de considérer le bien commun, non comme l'expression de normes universelles, mais comme « quelque chose qui est partagé par une communauté spécifique dans un contexte historique spécifique», où les interlocuteurs «demeurent intimement connectés à leurs biens et à leurs identités », et pour lesquels la réalisation du bien commun « ne consiste pas à transcender les différences [...] mais à veiller à l'intégration de tous ces biens qui sont constitutifs du moi et de l'identité des personnes impliquées » (163; ma traduction). Finalement, Blattberg juge que l'exigence d'égalité des chances ou des « libertés égales » n'est aucunement indispensable à la tenue d'une discussion authentique, que des interlocuteurs « radicalement inégaux », que ce soit en termes de richesse, de pouvoir ou 
d'aptitudes communicationnelles, peuvent parfaitement articuler des vérités et les faire partager aux autres, et que, en conséquence, le principe même d'égalité des chances ou des libertés égales devrait être accessible au débat et non figurer comme une exigence incontournable de la discussion ayant d'emblée une prééminence sur d'autres valeurs (160-161).

Mais Blattberg a aussi des reproches plus généraux à formuler à l'endroit de la démocratie délibérative, reproches dont dérivent en quelque sorte les critiques plus particulières que j'ai mentionnées et qui nous permettent de mieux en comprendre le sens. L'objection centrale qu'il adresse à cette approche est de s'appuyer sur une " théorie neutraliste de la conversation », c'est-à-dire de recourir à une "procédure théorique » (Et si nous dansions 44), ou à des règles qui « ont leur base dans la théorie, ce qui signifie qu'ils sont détachés de tout contexte pratique » (Blattberg, " Patriotic » 158 ; ma traduction). Habermas fait en effet valoir une version que l'on pourrait appeler procédurale de la démocratie délibérative en ce sens qu'il estime que le caractère raisonnable des résultats de la délibération démocratique dépend entièrement de la justesse (ou de l'impartialité) de la procédure de délibération, et que l'acceptabilité des résultats ne peut être évaluée à la lumière de critères de justice substantiels (par exemple, certains droits constitutionnels) indépendants de la procédure (L'intégration républicaine 316-327). Il s'agit là sans nul doute d'une affirmation controversée que n'endossent pas d'autres défenseurs de la démocratie délibérative. Mais Blattberg ne serait pas davantage satisfait si, en lieu et place d'une procédure neutre de discussion, la conversation patriotique qu'il a en vue était normée par des droits constitutionnels (Gutmann et Thompson 17), ou par une conception indépendante de la justice et de la raison publique (Rawls, " The Idea of Public Reason » et « The Idea of Public Reason Revisited »), conformément à une version plus substantielle de la démocratie délibérative. La raison en est que les droits, comme les procédures, font appel à ce que Blattberg (Et si nous dansions 30-31) nomme des " règles prescriptives ", c'est-à-dire à des normes contraignantes qui sont imposées de l'extérieur aux agents et auxquelles ils ont l'obligation de se conformer, à ces mêmes normes invoquées par la théorie neutraliste de l'État et de la justice de Pierre Elliott Trudeau et par l'approche «monarchiste » de la politique qu'il incarne, qui seraient les grandes responsables, selon Blattberg, du fait que nous nous sentions si peu chez nous au Canada (Si nous dansions). Or, une communauté républicaine fondée sur la conversation patriotique devrait plutôt faire appel à des « règles expressives », c'est-à-dire à des règles qui, au sens où l'entend Wittgenstein, sont intériorisées par les agents à partir du moment où ils savent comment en faire usage dans tel ou tel contexte (16-18). Ce savoir immanent des règles par l'usage suppose l'immersion dans une " forme de vie », et la participation à l'ensemble des pratiques, des traditions, des coutumes qui définissent une communauté historique. Bref, la conversation patriotique qu'envisage 
Blattberg n'aurait besoin, pour son bon fonctionnement, ni d'une procédure impartiale, ni de droits constitutionnels, encore moins d'une conception neutre de la justice ou de la raison publique, puisque ceux-ci sont « détachés de tout contexte pratique » (44). La seule chose requise des interlocuteurs serait une sensibilité au contexte, ainsi qu'une aptitude à s'en remettre à leur bon sens, à ces règles intériorisées par l'usage, à ces évidences préréflexives et à ce savoir d'arrière-plan qu'offre la forme de vie à laquelle ils appartiennent. En un mot : la conversation patriotique n'aurait à en appeler à rien d'autre qu'au « sens commun » (44), que Blattberg envisage comme « une forme interprétative du jugement qui doit être distinguée de la raison théorique en ce qu'elle est incompatible avec l'application à un contexte donné d'un ensemble systématique de règles formulé préalablement» (« Patriotic» 158; ma traduction), et qui « a beaucoup à partager avec l'esprit de la notion aristotélicienne de phronesis ou de prudence pratique » (164). La conversation patriotique, telle que l'envisage Blattberg, serait donc « de part en part herméneutique » (158).

\section{III}

Pour terminer mon analyse, $\mathrm{j}$ 'insisterai moins sur les critiques de Blattberg que sur la valeur de l'alternative qu'il propose. Je juge en effet qu'il n'est pas urgent de répondre de manière détaillée aux critiques de Blattberg, parce que la plupart d'entre elles sont bien connues et que les stratégies de réponse pour les contourner ont déjà été offertes et amplement discutées ${ }^{2}$. À titre d'exemple, l'humour, ou la flatterie, peuvent bien entendu contribuer à favoriser l'obtention de consensus, mais ce ne peut être que de manière accidentelle - Habermas parlerait sans doute dans leur cas d'« effets perlocutoires » possibles des actes de langage. Personne n'oserait envisager l'humour ou la flatterie comme une présupposition universelle de l'argumentation, contrairement par exemple à la sincérité des locuteurs, exigence qui, si elle est absente, nous autorise raisonnablement à mettre en doute la valeur du consensus obtenu, ce qu'on ne peut dire de l'humour ou de la flatterie. Il en va de même pour les autres exigences, comme la symétrie des interlocuteurs et l'égalité des chances qu'ils ont de faire valoir leurs points de vue. Remettre en question de telles exigences nous entraîne dans ce que K.-O. Apel appelle une " contradiction performative ", consistant, pour le locuteur, à n'avoir d'autre choix que de présupposer ce qu'il conteste pour que son objection ait un sens, tant pour lui-même que pour les interlocuteurs (918-921). En contestant l'idée que la symétrie ou l'égalité constitue une exigence universelle du dialogue, le locuteur doit inévitablement supposer, s'il veut que son objection soit véritablement entendue, qu'il occupe une position symétrique à tous les autres interlocuteurs, ou qu'il dispose d'une chance équivalente de formuler ses arguments.

Les critiques plus générales de Blattberg reprennent, de leur côté, de nombreuses critiques récentes formulées à l'intérieur même des rangs de 
la démocratie délibérative. Plusieurs démocrates délibératifs estiment en effet que les versions initiales proposées de la démocratie délibérative par ses théoriciens majeurs (Habermas au premier chef) seraient irréalistes. Pour certains (Valadez 58-67; Young 52-80; Fish 92-93; Simon 50-52; Dryzeck 58), les contraintes de neutralité ou d'impartialité imposées à la délibération pour assurer la justesse des résultats seraient trop exigeantes et risquent d'exclure du débat public plusieurs groupes de citoyens incapables d'exprimer adéquatement leurs intérêts dans un tel cadre, comme les minorités culturelles et religieuses. Pour d'autres (Bohman 71-105; McCarthy 121-128; Weinstock; Devaux 97-107), la recherche de consensus autour d'intérêts universalisables constituerait un idéal beaucoup trop élevé si l'on tient compte de la diversité des valeurs et du caractère irréconciliable des visions du monde qui forment un trait marquant de nos sociétés pluralistes. Pour eux, la recherche de " compromis moraux », voire la valorisation de la négociation et de la dimension proprement stratégique de la délibération, constitueraient des objectifs beaucoup plus réalistes et davantage appropriés à nos sociétés pluralistes. Blattberg verrait sans doute dans ces orientations récentes empruntées par les démocrates délibératifs un certain penchant pour l'approche polyarchiste de la politique qu'il condamne. Elles partagent néanmoins avec son approche patriotique un objectif analogue : rendre la pratique délibérative plus sensible au contexte et à la diversité des perspectives interprétatives des délibérants, ce qui implique d'assouplir, voire de relativiser les normes du discours. Je dirais cependant que ce qui distingue fondamentalement la conversation patriotique de Blattberg de ces orientations récentes empruntées par les démocrates délibératifs n'est pas tant l'objectif poursuivi que les positions philosophiques qui lui servent d'arrière-plan. Ces positions sont celles que font valoir plusieurs courants de la philosophie contemporaine, comme la philosophie herméneutique, le néo-aristotélisme, l'éthique de la vertu, voire le communautarisme, positions que je qualifierais globalement d'anti-théoriques, de particularistes ou de contextualistes. En endossant ce genre de positions, je crains que l'approche patriotique de Blattberg ne fasse que substituer aux difficultés de l'approche délibérative d'autres difficultés, peut-être plus grandes, auxquelles l'approche délibérative s'est précisément efforcée de proposer des pistes de solution.

L'un des problèmes les plus urgents qu'ont tenté de résoudre, non seulement les démocrates délibératifs, mais la majorité des philosophes politiques contemporains, est celui du pluralisme : comment permettre à une diversité de groupes dont les membres partagent des croyances et des valeurs irréconciliables de cohabiter ensemble de manière pacifique? La théorie de la justice de Rawls, mettant en avant l'idée d'un « consensus par recoupement» autour de certaines normes constitutionnelles minimales faisant office de « raison publique », s'offre sans doute comme l'une des approches les plus influentes et les plus discutées à l'heure actuelle. Le patriotisme constitutionnel 
de Habermas, joint à sa conception délibérative de la démocratie, offre une autre avenue que plusieurs ont empruntée pour résoudre le problème de la diversité. Mentionnons également les travaux de Kymlicka, qui propose l'idée d'une citoyenneté différenciée selon les groupes culturels et d'un système de droits visant à protéger les minorités. Or, toutes ces approches ont pour point commun d'en appeler, pour reprendre le langage de Blattberg, à des « règles prescriptives » (à des normes constitutionnelles, à des droits, à des procédures, etc.), solution qu'il rejette. Toutes, également, s'en remettent à l'idée d'un "bien commun » défini de la manière la plus minimaliste possible (s'articulant autour de principes constitutionnels, d'une procédure de délibération, ou, comme chez Kymlicka, de biens culturels « minces » comme une langue publique et des institutions publiques communes), ce qui ne peut non plus satisfaire Blattberg. Mais la question se pose alors de savoir comment, dans les conditions pluralistes propres aux démocraties libérales actuelles et, en particulier, à un pays comme le Canada, il est possible de s'en remettre à l'idée d'un « bien commun » plus substantiel défini à partir de règles « expressives», d'un " sens commun » inhérent à une forme de vie particulière, de valeurs patriotiques communes, bref, à ce qui semble plutôt se présenter comme la recherche d'un idéal perfectionniste d'excellence, de vertu ou de vie bonne qu'une conversation sincère entre les membres d'une communauté républicaine permettrait de cimenter. Si c'est en cela que consiste la politique du bien commun que Blattberg a à offrir pour répondre aux maux qui affligent le Canada et qui font que nous nous sentions si peu chez nous dans le paysage constitutionnel de notre pays, si ce sont là les conditions devant permettre le rapprochement des différentes communautés nationales dont se compose le pays, je doute fort que de telles conditions soient un jour réunies, et on peut se demander si elles ne sont pas en bout de piste plus utopiques et irréalistes que les conditions idéales de délibération démocratique que Blattberg dénonce.

\section{Notes}

1. Cette localisation est bien entendu discutable. Un État ne peut se passer de politiques sur les langues officielles et sur la langue de la vie publique. Comme l'a fait valoir de manière convaincante Kymlicka (111), une stricte séparation de l'État et de la langue ou de la culture est une entreprise à toute fin pratique impossible, contrairement à la séparation de l'État et de l'Église. Pour des raisons d'espace, je laisserai cette discussion de côté pour le moment.

2. Voir par exemple Thompson \& Held. 


\section{Bibliographie}

Apel, Karl-Otto. «La question de la fondation ultime de la raison.» Critique. Vingt ans de pensée allemande 413 (1981): 895-928.

Blattberg, Charles. «Patriotic, Not Deliberative, Democracy.» Critical Review of International Social and Political Philosophy 6.1(2003) : 155-174. Et si nous dansions? Pour une politique du bien commun au Canada. Montréal : Les Presses de l’Université de Montréal, 2004.

Bohman, James. Public Deliberation : Pluralism, Complexity, and Democracy. Cambridge, MA : MIT Press, 1996.

Deveaux, Monique. Gender and Justice in Multicultural States. Oxford : Oxford University Press, 2006.

Dryzeck, John. Deliberative Democracy and Beyound : Liberals, Critics, and Contestations. Oxford : Oxford University Press, 2000.

Fish, Stanley. «Mutual Respect as a Device of Exclusion.» Deliberative Politics : Essays on Democracy and Disagreement. Éd. Stephen Macedo. Oxford : Oxford University Press, 1999. 88-102.

Gutmann, Amy \& Thompson, Dennis. Democracy and Disagreement. Cambridge, MA : Cambridge University Press, 1996.

Habermas, Jürgen. Morale et communication. Conscience morale et activité communicationnelle. Paris : Les Éditions du Cerf, 1986. L'intégration républicaine. Paris : Fayard, 1998.

Kymlicka, Will. Multicultural Citizenship. Oxford : Oxford University Press, 1995.

McCarthy, Thomas. «Legitimacy and Diversity : Dialectical Reflections on Analytical Distinctions.» Habermas on Law and Democracy : Critical Exchanges. Éds. A. Arato \& M. Rosenfeld. California : University of California Press, 1998. 115-153.

Rawls, John. «The Idea of Public Reason.» Political Liberalism. John Rawls. New York : Columbia University Press, 1993. 212-254. «The Idea of Public Reason Revisited.» The Law of Peoples. John Rawls. Cambridge, MA : Harvard University Press, 1999. 129-180.

Simon, William H. «Three Limitations of Deliberative Democracy : Identity Politics, Bad Faith, and Indeterminacyé.» Deliberative Politics : Essays on Democracy and Disagreement. Éd. Stephen Macedo. Oxford : Oxford University Press, 1999. 49-57.

Thompson, John B. \& Held, David (Éds.). Habermas : Critical Debates. Cambridge, MA : The MIT Press, 1982.

Valadez, Jorge. Deliberative Democracy, Political Legitimacy, and SelfDetermination in Multicultural Societies. Boulder, CO : Westview Press, 2001.

Weinstock, Daniel. «Is 'Identity' a Danger to Democracy ?» Identity, Self-Determination, and Secession. Éds. I. Primoratz \& A. Pavkovic. Aldershot : Ashgate Publishing, 2006.

Young, Iris Marion. Inclusion and Democracy. Oxford :

Oxford University Press, 2000. 